\title{
The Cross Sectional Study on the Association between Sexual Awareness and Cognitive Social Capital among High School Students in Ho Chi Minh City, Vietnam
}

\author{
Kaori Watanabe \\ National College of Nursing, Japan \\ Email: kaori.w@me.com
}

Received 1 July 2016; accepted 17 July 2016; published 20 July 2016

Copyright (C) 2016 by author and Scientific Research Publishing Inc.

This work is licensed under the Creative Commons Attribution International License (CC BY). http://creativecommons.org/licenses/by/4.0/

(c) (i) Open Access

\begin{abstract}
Purpose: We aimed to reveal the association between cognitive social capital and sexual awareness among high school students in Ho Chi Minh City, Vietnam, where adolescent premarital sex is considered to be wide-spreading as consequence of socioeconomic changes, in comparison to our previous study in Hanoi, 2012. Methods: We conducted a questionnaire-based survey and a cross sectional study in late 2014, with 2051 eleventh graders at three high schools in Ho Chi Minh City. Complete data was received from 1935 respondents (94.3\%). We assessed basic attributes, sexual knowledge, sexual awareness, sexual experience of peers, cognitive social capital such as a sense of community belonging, and Rosenberg Self-Esteem score. After the independent $t$-test and multiple logistic regression analyses on sexual awareness were performed assigning trivalent Rosenberg Self-Esteem score, sexual knowledge, and sexual experience of peers as independent variables (model 1), followed by analysis adjusted for cognitive social capital (model 2). This study was approved by Japanese Red Cross Akita College of Nursing (No. 24-003). Results: 0 1935 respondents, $99.9 \%$ were 16 or 17 years old and mostly from nuclear families $(71.1 \%)$. Of all participants, $45.8 \%$ in males and $49.4 \%$ in females correctly answered on sexual knowledge, $47.6 \%$ of males and $28.3 \%$ of females accepted premarital sex, $60.0 \%$ of male and $65.0 \%$ of females knew peers with sexual intercourse experience, and $78.1 \%$ of males and $79.1 \%$ of females agreed with a sense of community belonging. When multiple logistic regression analyses performed, in model 1 , sexual awareness was significantly associated with Rosenberg Self-Esteem score in female. In model 2, above association disappeared but significant association was found between sexual awareness and cognitive social capital in both sexes OR (95\% CI), male 1.39 (1.05 - 1.84), female 1.99 (1.49 - 2.65). Discussion: The conservative traditional culture in both Hanoi and Ho Chi Minh City seems to be similar to the concept of cognitive social capital and it is considered to have influenced sexual awareness of high school students in Hanoi and Ho Chi Minh City, Vietnam. Conclusion: Cognitive social capital is significantly associated with sexual awareness among high school students in urban areas of Vietnam.
\end{abstract}

How to cite this paper: Watanabe, K. (2016) The Cross Sectional Study on the Association between Sexual Awareness and Cognitive Social Capital among High School Students in Ho Chi Minh City, Vietnam. Advances in Sexual Medicine, 6, 33-39. 
Keywords

\section{Cognitive Social Capital, Sexual Behavior, Sexual Awareness, Self-Esteem, Adolescent, High School Student, Ho Chi Minh City, Vietnam}

\section{Introduction}

Adolescent sexual behavior, particularly premarital sex, is a growing public health concern around the world because of adolescent pregnancy and sexually transmitted diseases as its outcome. In many circumstances, pregnant adolescent girls face a much higher risk of maternal mortality as their immature bodies are unable to cope with the experience [1]. Another serious problem is unsafe abortion, which often causes the death of many adolescent girls and injures many more [2] [3]. Likewise in Vietnam, premarital or unprotected sexual behavior is a growing health and social concern. The country's dramatic economic growth and political changes have resulted in social change, including premarital sexual attitudes and behaviors. Although premarital sex is longconsidered a social taboo, the government has branded it a "social evil" and believes that high rates of premarital sexual activities are widespread among the younger generation [4]. Along with structural economic and political changes resulting from the country's renovation, the increased influential impact of foreign media sources such as the Internet, imported movies, pornography, and sexualized music that Vietnamese youth are exposed to is often mentioned as a leading cause of unhealthy social changes [4]-[6].

According to a Survey Assessment of Vietnamese Youth (SAVY), the largest and most comprehensive survey of youth in Vietnam, the mean age a youth (aged 14 to 25 years) first experiences sexual intercourse had decreased from 19.6 years old in 2003 to 18.1 years old in 2008, and the rate of premarital sex increased from $7.6 \%$ in 2003 to $9.5 \%$ in 2008 [7] [8]. Ghuman et al.'s research (2006) also reported that premarital sex was becoming more common in Vietnam [9].

Sexual activity is often initiated between the ages of 15 and 22 [10], when many adolescents are still at school days, and more than half (51.2\%) of new HIV infections are among 15 to 24 year olds [11]. Therefore, it is necessary to address the sexual behaviors of high school students. A considerable number of demographic and reproductive health studies in Vietnam have been published in recent years [10]. Of these studies, one study focused on HIV/AIDS preventive behavior [12], and most previous studies have focused on raising self-esteem and sex education as important intervention techniques that influence sexual awareness and behavior of adolescents [13]. In their systematic review of fifteen studies, McPherson et al. explored the role and impact of social capital on sexual health outcomes [14]-[20]. The evidence suggested that social capital is a protective factor for sexual health behaviors [20] [21].

Meanwhile, Watanabe et al. report in research in Hanoi that previous theory that sexual knowledge and selfesteem are determinants of sexual awareness has been negated as persons who are permissive to premarital sex are recognized to show higher cognitive social capital such as a sense of community belonging and contribution to society when several factors such as gender, self-esteem, sexual knowledge, sexual experience of peers (siblings and friends) are considered simultaneously [22] [23]. Based on reports showing that low-levels of cognitive social capital increased the risk of unhealthy sexual behavior [20], we hypothesized that the sexual attitudes of Vietnamese high school students' permissiveness and acceptance of premarital sex, are influenced by cognitive social capital, such as a sense of community belonging. Thus, the purpose of this cross-sectional study was to examine whether cognitive social capital is associated with raised awareness of sexual behaviors among Vietnamese high school students. Also, we aimed to clarify determinants for sexual awareness among high school students in urban area of Vietnam by verifying that if we can acquire the same results from this research in Ho Chi Minh City in South part of Vietnam which is different in cultural background from Hanoi, the Capital city in North part of Vietnam, where Watanabe et al. researched [22].

\section{Methods}

The participants of this study were randomly (but gender and school equally-balanced) selected from eleventh grade students at three public high schools in Ho Chi Minh City (area: $2096 \mathrm{~km}^{2}$, population: 7992 million), the southern metropolitan city of the Socialist Republic of Vietnam (area: 330,967 km², population 90,729 million) 
[Vietnam General Statistics Office-GSO, 2014] [24]. The Center for Population Information and Documentation (CPID), the General Office for Population and Family Planning (GOPFP), and the Ministry of Health of Vietnam supported the survey. We conducted a questionnaire-based survey from October to November, 2014, with 2051 students participating. Responses were received from 2051 students, (a response rate of 100\%). Incomplete surveys such as no description on gender and age were excluded from the analysis. Complete data was received from 1935 respondents (94.3\%), consisting of 933 males (48.2\%) and 1002 females (51.8\%).

The survey was a self-administrated questionnaire which is the same questionnaire previously made and used for high school students in Hanoi (Watanabe et al. 2014) [22] [23]. We obtained permission to conduct the survey from the appropriate person in charge at each school. The high school staff and GOPFP distributed the questionnaires, and explained the details to participating students. The survey was anonymous. Each student sealed the completed survey in an envelope to protect their privacy.

The questionnaire assessed the participants' sexual knowledge, awareness of sexual behavior, awareness of peers' sexual intercourse experience, cognitive social capital, and used Rosenberg Self-Esteem Scale Score (SE Score) [25]. We assigned each variable to groups such as (1) male or female for gender, (2) whether the respondent agreed or not with the questions on social capital and sexual awareness, or (3) correct or incorrect answers to the sexual knowledge questions. Then, we compared the means of the two groups using the independent $t$-test, and performed multiple logistic-regression analysis to examine the association between acceptance of premarital sex and social capital with adjustment for Rosenberg Self-Esteem score (divided into three groups with the mean value and standard deviation), timing to wear a condom, and sexual experience of peers (model 1). This was followed by analysis of the associations adjusted for sense of community belonging, Rosenberg Self-Esteem score, wearing a condom, and sexual experience of peers (model 2).

All analyses were computed using SPSS21 (Chicago, IL) statistical software.

This study was approved by the Ethical Review Board of the Japanese Red Cross Akita College of Nursing (approval number 24-003). We had the cooperation of the high schools and obtained written informed consent from the participating students.

\section{Results}

Of the 1935 respondents with complete data, $99.9 \%$ was 16 or 17 years old. Most participants $(71.1 \%)$ were from the nuclear families.

Table 1 shows the sexual knowledge of the participants. The percentage of the participants who correctly answered the question on the proper timing to wear a condom was $45.8 \%$ in males and $49.4 \%$ in females. Table 1 also shows the results of sense of community belonging and awareness of sexual behavior. Of all participants,

Table 1. Sexual awareness, sexual knowledge, sense of community belonging.

\begin{tabular}{|c|c|c|}
\hline \multirow{2}{*}{ Variable } & Male & Female \\
\hline & n (\%) & n (\%) \\
\hline \multicolumn{3}{|c|}{ Question about timing to wear condom $(\mathrm{n}=1935)$} \\
\hline Correct answer & $427(45.8)$ & $495(49.4)$ \\
\hline Incorrect answer & $506(54.2)$ & $507(50.6)$ \\
\hline \multicolumn{3}{|c|}{ Permissiveness toward premarital sex $(n=1926)$} \\
\hline Permissible & $486(52.4)$ & $716(71.7)$ \\
\hline Impermissible & $442(47.6)$ & $282(28.3)$ \\
\hline \multicolumn{3}{|c|}{ Sexual experience of peers (friends and siblings) $(n=1890)$} \\
\hline Yes & $548(60.0)$ & $634(65.0)$ \\
\hline No & $366(40.0)$ & $342(35.0)$ \\
\hline \multicolumn{3}{|l|}{ Sense of community belonging $(\mathrm{n}=1932)$} \\
\hline Agree & $729(78.1)$ & $790(79.1)$ \\
\hline Disagree & 204 (21.9) & 209 (20.9) \\
\hline
\end{tabular}


$47.6 \%$ of males and $28.3 \%$ of females accepted premarital sex, and $60.0 \%$ of male and $65.0 \%$ of females knew peers with sexual intercourse experience. As to cognitive social capital, $78.1 \%$ of males and $79.1 \%$ of females agreed with a sense of community belonging.

Table 2 shows the Rosenberg Self-Esteem Scale scores. The mean value of the Rosenberg Self-Esteem score was $21.5 \pm 2.6$ and female scores were significantly higher than male scores $(P<0.01)$. This was similar to average scores in Western countries [25], but different from the result of eleventh graders of high schools in Hanoi that the overall mean score was $18.0 \pm 3.9$ and male scored higher than females. There was no significant difference in Rosenberg Self-Esteem score depending on the presence or absence of sexual experience, sexual knowledge, sexual experience of peers, and whether permissible or impermissible to premarital sex or not.

Table 3 shows the results of the multiple logistic regression analyses, including sense of community belonging as a variable. There was a positive association between sense of community belonging and acceptance of premarital sex even after adjusting for Rosenberg Self-Esteem score, sexual knowledge, and sexual experience of peers. In model 1 , the acceptance of premarital sex was significantly associated with Rosenberg Self-Esteem score and sexual experience of peers. In model 2, after adjusting for a sense of community belonging, the association disappeared between acceptance of premarital sex and Rosenberg Self-Esteem score, and sexual experience of peers, but a significant association was found between acceptance of premarital sex and a sense of community belonging OR (95\%CI), male 1.39 (1.05 - 1.84), female 1.99 (1.49 - 2.65).

\section{Discussion}

Our results indicate that sexual knowledge, sexual experience of peers and Rosenberg Self-Esteem score are explanatory factors relevant to the risk of sexual behaviors when a sense of community belonging is not included in a logistic regression model. This coincides with previous research that reported adequate sexual knowledge, lesser sexually experienced peers around, and high level of self-esteem were factors associated with the risk of adolescent sexual behaviors. However, when a sense of community belonging was included in the logistic regression model, the association was attenuated, and we found only a sense of community belonging significantly associated with the risk of sexual behaviors. This implies that, at least among adolescents in developing countries like Vietnam, cognitive social capital is a more important factor associated with the risk of sexual behaviors compared with sexual knowledge, sexual experience of peers and self-esteem.

This finding is in line with a considerable number of studies on the association between cognitive social capital and the risk of sexual behaviors published in the US or other countries [14]-[20]. These concluded that cognitive social capital decreases the risk of sexual behaviors.

Table 2. Rosenberg self-esteem scale scores.

\begin{tabular}{|c|c|c|c|c|c|c|}
\hline \multirow{2}{*}{ Variable } & \multicolumn{3}{|c|}{ Male } & \multicolumn{3}{|c|}{ Female } \\
\hline & $\mathrm{n}$ & mean(SD) & $P$ value & $\mathrm{n}$ & mean(SD) & $P$ value \\
\hline \multicolumn{7}{|l|}{ Sexual experience } \\
\hline Presence & 38 & $21.2(2.6)$ & \multirow{2}{*}{0.6} & 15 & $21.4(2.1)$ & \multirow{2}{*}{0.8} \\
\hline Absence & 822 & $21.4(2.8)$ & & 926 & $21.6(2.5)$ & \\
\hline \multicolumn{7}{|l|}{ Premarital sex } \\
\hline Permissible & 456 & $21.4(2.7)$ & \multirow{2}{*}{0.7} & 686 & $21.6(2.5)$ & \multirow{2}{*}{0.9} \\
\hline Impermissible & 416 & $21.5(2.9)$ & & 269 & $21.6(2.5)$ & \\
\hline \multicolumn{7}{|c|}{ Sexual knowledge (Propertiming to wear condom) } \\
\hline Correct answer & 402 & $21.4(2.7)$ & \multirow{2}{*}{0.6} & 478 & $21.5(2.4)$ & \multirow{2}{*}{0.7} \\
\hline Incorrect answer & 475 & $21.5(2.8)$ & & 481 & $21.6(2.5)$ & \\
\hline \multicolumn{7}{|l|}{ Sexual experience of peers } \\
\hline Yes & 343 & $21.5(2.7)$ & \multirow{2}{*}{0.4} & 323 & $21.8(2.4)$ & \multirow{2}{*}{0.1} \\
\hline No & 516 & $21.4(2.9)$ & & 615 & $21.5(2.5)$ & \\
\hline
\end{tabular}


Table 3. Results of logistic regression analysis on the association between premarital sex permissiveness and sense of community belonging.

\begin{tabular}{|c|c|c|c|c|c|c|c|c|}
\hline \multirow{3}{*}{ Variables } & \multicolumn{4}{|c|}{ Model 1} & \multicolumn{4}{|c|}{ Model 2} \\
\hline & Male & & Female & & Male & & Female & \\
\hline & OR (95\%CI) & $P$ & OR (95\%CI) & $P$ & OR (95\%CI) & $P$ & OR (95\%CI) & $P$ \\
\hline
\end{tabular}

Sense of Community

Belonging
Agree
$1.39(1.05-1.84) \quad 0.02 \quad 1.99(1.49-2.65) \quad<0.01$
Disagree
ref
ref

Self-Esteem Scale Score
$24<$
$1.37(1.08-1.73) \quad 0.01$
$2.65(2.04-3.43)$
$<0.01$
$1.15(0.87-1.52)$
$0.33 \quad 1.82(1.34-2.48)$
0.25
$20-24$
$1.12(0.73-1.72) \quad 0.6$
$2.21(1.34-3.62)$
0.02
0.98 (0.63 - 1.52)
$0.91 \quad 1.56(0.92-2.63)$
0.1
$<20$
ref
ref
ref
ref

Sexual knowledge

(Proper timing

to wear condom)

Correct answer

$0.86(0.66-1.12) \quad 0.28$

$1.06(0.81-1.39)$

0.67

$0.82(0.63-1.07)$

0.15

$0.99(0.75-1.30)$

0.95

Incorrect answer

ref

ref

ref

ref

Most peer have sexual experience

Not applicable

$0.64(0.47-0.87) \quad 0.04 \quad 1.99(1.08-3.67)$

0.03

$0.84(0.60-1.16)$

0.29

$0.86(0.61-1.21)$

0.38

Applicable

ref

ref

ref

ref

OR: Odds Ratio, CI: Confidence Interval. Results are based on logistic regression analysis. Model 1 has been adjusted for Rosenberg Self-Esteem Scale Score, Proper Timing to Wear Condom and Most of Peer Have Sexual Experience. Model 2 has been adjusted for Community Belonging, Rosenberg Self-Esteem Scale Score, Proper Timing to Wear Condomand Most of Peer Have Sexual Experience.

In Vietnam, there is deep-rooted culture that people respect to belonging community, family, and the elders and follow the teachings of them [26] [27]. It suggests that, regardless South or North, it coincide with the perception of Watanabe et al. saying that these concepts seem to be similar to the concept of cognitive social capital in the western world [25].

Prevalence of sexual coitus among adolescents and young adults was lowest in Ho Chi Minh City on a par with the report on Hanoi by Watanabe et al. [21], and Vietnamese adolescents with more traditional views were less likely to engage in sexual activity [27]. This finding suggested that the conservative traditional culture may have a distinct association with adolescents' sexual behaviors, and that cognitive social capital, in general, is associated with less risky sexual behavior [20] [21]. Also, throughout Vietnam, the conservative traditional culture regards virginity as a virtue among both males and females [5]. Cultural environment change is an important factor influencing adolescents' sexual behaviors. In the wake of modernization, rapid industrialization, and contemporary globalization, Vietnam is undergoing marked sociocultural changes, which are weakening aspects of the conservative traditional culture, including dependence and connectedness to the family and community.

A possible reason that sexual knowledge and self-esteem were weaker factors than a sense of community belonging, compared with previous studies, may be that this study simultaneously analyzed sexual knowledge, sexual experience of peers and self-esteem with cognitive social capital.

There is a major difference in culture and historic background between South and North in Vietnam. Since this research obtained the same results of the research of Hanoi in North of Vietnam by Watanabe et al. (2014) [22], we may conclude that determinants of sexual awareness, at least in concerning high school students in urban areas of Vietnam, are different from above mentioned previous findings such as sexual knowledge and self-esteem. This implies that cognitive social capital is a more important determinant factor of sexual awareness compared with any other factors. Therefore, future studies of adolescent sexual behaviors should consider the 
cognitive social capital factors.

The findings of this study highlight the importance of strengthening cognitive social capital such as a sense of community belonging as well as sexual education to enrich reproductive health knowledge among high school students in urban areas of Vietnam like Ho Chi Minh City and Hanoi as Vietnamese conservative traditional culture still appear to have a strong influence on their attitudes about premarital sex while urban youth are beginning to be easing of judgement and accepting of premarital sex. In other words, increasing opportunities of participating in community events and volunteer activities for high school students along with sexual education at school is useful approach to enhance cognitive social capital to prevent them from risky sexual behaviors.

Several study limitations need to be considered. First, the cross-sectional nature of this survey design does not allow for changes in participants' beliefs, past, present and future, to be examined. This can be achieved with a long-term longitudinal study. Second, because this is a self-administered questionnaire survey, there is a possibility of reporting bias.

Since this research and the research of Hanoi in 2014 by Watanabe et al. [22] were both targeted to high school students in urban areas, hereafter it is necessary to clarify the whole picture of adolescents by researching on high school students in rural area and adolescents who do not move up to high school.

\section{Conclusion}

Cognitive social capital, such as a sense of community belonging, was found to be significantly associated with sexual awareness such as a negative attitude towards premarital sex among high school students in urban areas of Vietnam. This suggests that the concept of cognitive social capital should be enhanced in sexual health promotion and education especially with adolescents.

\section{Acknowledgements}

We are grateful for the collaboration of staff members from the Ministry of Health Center for Population Information and Documentation (CPID), General Office for Population and Family Planning (GOPFP) of Socialist Republic of Vietnam, and participating high school staff members, students, and their parents/guardians in conducting this study.

\section{References}

[1] Anthony, D. and United Nations Children’s Fund (UNICEF) (2011) The State of the World's Children 2011Adolescence: An Age of Opportunity. http://www.unicef.org/sowc2011/pdfs/SOWC-2011-Main-Report_EN_02092011.pdf

[2] UNFPA (2013) The State of World Population 2013 Motherhood in Childhood—Facing the Challenge of Adolescent Pregnancy.

[3] World Health Organization (WHO) (2008) Unsafe Abortion: Global and Regional Estimates of the Incidence of Unsafe Abortion and Associated Mortality in 2008. 6th Edition, WHO, Geneva.

[4] Jayakody, R., Heckert, J. and Anh, D. (2010) Social Change and Premarital Sexual Behavior and Attitudes in Vietnam. SAGE Publications Ltd., Thousand Oaks. http://dx.doi.org/10.4135/9781446252161.n11

[5] Linh, L. and Blum, R. (2009) Premarital Sex and Condom Use among Never Married Youth in Vietnam. International Journal of Adolescent Medicine and Health, 21, 299-312.

[6] Ngo, A., Ross, M. and Ratliff, E. (2008) Internet Influence on Sexual Practice among Young People in Hanoi, Vietnam. Culture, Health \& Sexuality, 10, S201-S213. http://dx.doi.org/10.1080/13691050701749873

[7] Ministry of Health, General Statistic Office, World Health Organization and the United Nations Children Fund (2005) Survey Assessment of Vietnamese Youth Round 1 (SAVY 1). Hanoi.

[8] Ministry of Health, General Statistic Office, World Health Organization and the United Nations Children Fund (2010) Survey Assessment of Vietnamese Youth Round 2 (SAVY 2). Hanoi.

[9] Ghuman, S., Loi, V., Huy, V. and Knodel, J. (2006) Continuity and Change in Premarital Sex in Vietnam. International Family Planning Perspectives, 32, 166-174. http://dx.doi.org/10.1363/3216606

[10] Mensch, B.S., Clark, W.H. and Dang, A.N. (2002) Premarital Sex in Vietnam: Is the Current Concern with Adolescent Reproductive Health Warranted? Policy Research Division, Population Council No. 163. http://www.popline.org/node/245619

[11] van Wijingaarden, J. (2006) UNICEF Consultant Exploring Factors and Processes Leading to HIV Risk among the Most Vulnerable Children and Adolescent in Vietnam. 
[12] Taechaboonsermsak, P., Tuan, L. and Apinuntavech, S. (2008) Factors Associated with HIV/AIDS Preventive Behavior among High School Students in Dongda District, Hanoi, Vietnam. Journal of Public Health, 38, 174-185.

[13] Goodson, P., Buhi, E.R. and Duncsmore, S. (2006) Self-Esteem and Adolescent Sexual Behaviors, Attitudes, and Intentions: A Systematic Review. Journal of Adolescent Health, 38, 310-319. http://dx.doi.org/10.1016/j.jadohealth.2005.05.026

[14] McPherson, K., Kerr, S., McGee, E., Cheater, F. and Morgan, A. (2013) The Role and Impact of Social Capital on the Health and Wellbeing of Children and Adolescents: A Systematic Review. Glasgow Centre for Population Health. http://www.gcph.co.uk/assets/0000/3647/Social_capital_final_2013.pdf

[15] Harris, L. (2007) Association between Youth Assets and Sexual Activity: Does Adult Supervision Play a Role? Child Care Health and Development, 33, 448-454. http://dx.doi.org/10.1111/j.1365-2214.2006.00695.x

[16] Daniel, W. (2006) Parental Influence on Young People’s Sexual Behavior: A Longitudinal Analysis. Journal of adolescence, 29, 474-494.

[17] Oman, R. (2005) Youth Assets and Sexual Risk Behavior: The Importance of Assets for Young Residing in One-Parent Households. Perspectives on Sexual and Reproductive Health, 37, 25-31. http://dx.doi.org/10.1363/3702505

[18] Evans, A. (2004) An Exploration of the Relationship between Youth Assets and Engagement in Risky Sexual Behaviors. Journal of Adolescent Health, 35, 424.e21-424.e30. http://dx.doi.org/10.1016/S1054-139X(04)00073-4

[19] Oman, R., Vesely, S., et al. (2004) The Association between Multiple Youth Assets and Sexual Behavior. American Journal of Health Promotion, 19, 12-18. http://dx.doi.org/10.4278/0890-1171-19.1.12

[20] Crosby, R. (2003) Social Capital as a Predictor of Adolescents' Sexual Risk Behavior: A State-Level Exploratory Study. AIDS and Behavior, 7, 245-252. http://dx.doi.org/10.1023/A:1025439618581

[21] Agardh, A., et al. (2010) Social Capital and Sexual Behavior among Ugandan University Students. Global Health Action, 3, 5432. http://dx.doi.org/10.3402/gha.v3i0.5432

[22] Watanabe, K., Kaneko, Y., Fujita, K. and Motohashi, Y. (2014) The Association between Awareness of Sexual Behavior and Cognitive Social Capital among High School Students in Vietnam. Open Journal of Preventive Medicine, 4, 116-122. http://dx.doi.org/10.4236/ojpm.2014.43016

[23] Watanabe, K., Saruta, R. and Kato, N. (2014) Sources of Sexual Knowledge among Vietnamese High School Students. Advances in Reproductive Sciences, 2, 83. http://dx.doi.org/10.4236/arsci.2014.24010

[24] General Statistic Office of Vietnam, Statistical Year Book 2014. https://www.gso.gov.vn/Default_en.aspx?tabid=491

[25] Rosenberg, M. (1965) Society and the Adolescent Self-Image. Princeton University Press, Princeton, NJ. http://www.yorku.ca/rokada/psyctest/rosenbrg.pdf http://dx.doi.org/10.1515/9781400876136

[26] Dalton, R., et al. (2002) Social Relations and Social Capital in Vietnam: The 2001 World Values Survey. Comparative Sociology, 1, 369-386.

[27] Gao, E., Zuo, X., Wang, L., Lou, C., Cheng, Y. and Zabin, L. (2012) How Does Traditional Confucian Culture Influence Adolescents' Sexual Behavior in Three Asian Cities? Journal of Adolescent Health, 50, S12-S17. http://dx.doi.org/10.1016/j.jadohealth.2011.12.002

\section{Submit or recommend next manuscript to SCIRP and we will provide best service for you:}

Accepting pre-submission inquiries through Email, Facebook, LinkedIn, Twitter, etc.

A wide selection of journals (inclusive of 9 subjects, more than 200 journals)

Providing 24-hour high-quality service

User-friendly online submission system

Fair and swift peer-review system

Efficient typesetting and proofreading procedure

Display of the result of downloads and visits, as well as the number of cited articles

Maximum dissemination of your research work

Submit your manuscript at: http://papersubmission.scirp.org/ 